\title{
Factors Influencing Media Choice for Interact with Their Students among Lectures of Two Academic Institutions: Case of Iran
}

\author{
Dr. Abbas Ghanbari Baghestan (Corresponding author) \\ Department of Communication, Universiti Putra Malaysia \\ Po box 43400, Serdang, Selongor, Malaysia \\ Tel: 60-1-2274-8576 E-mail: ghanbari.abbas@gmail.com
}

Muhammad Rahmati

Sociology, Shahed University, Iran

E-mail: Rahmatim63@yahoo.com

Musa Abu Hassan

Department of Communication, Universiti Putra Malaysia

Po box 43400, Serdang, Selongor, Malaysia

E-mail: musa@putra.upm.edu.my

\begin{abstract}
The aim of this paper is to report the findings on the impact of communication technology as channels on interaction between academic staff and their students, conducted at an Iranian higher learning institution. Study focused on media choice and it attempted to determine the communication channels most used by academic staffs in interacting with their students and the reasons they chose these channels. It also intended to find out whether there was a significant relationship between communication channels most used by academic staffs and their perception of media richness. The results revealed that though there is the existence of new communication technologies such as the internet which offers faster and cheaper facilities, Face-to-Face communication is the most used and preferred communication channel by academic staffs in interacting with their students. In addition there was significant relationship between communication channel most used by respondents and their perception of media richness and social presence. That's why with the higher level of social presence, their level of experience with a channel increases. The findings of this study extend two of the most widely investigated media choice theories: Media Richness Theory (MRT) and Social Presence Theory (SPT).
\end{abstract}

Keywords: Computer Mediated Communication, Face to face communication, Media Richness Theory, Social Presence Theory

\section{Introduction}

Although face to face meetings are still likely to be an important channel, however, with the growth of new communication technology, it is no longer the sole communication medium used by academic staffs in interaction with their students. New communication technologies that have emerged in recent years, offer new channels and made possible new and expanded forms of communicating. These technologies as a new media have become an integral component of organizational communication as they are more convenient and less expensive than traveling to face-to-face meetings as well as being integrated into multi-media environments and digital networks (Baltes et al., 2002).

New communication technologies and its facilities (such as e-mail, voice-mail and video conferencing over digital networks) have come to be known as the new media as opposed to the traditional media of face-to-face meetings, telephone and text based documents. Thus, research into understanding factors influencing communication media 
Choice and exploring optimal ways of communicating has sparked the interest of academics and practitioners alike. There has been much research exploring the use of the new media attempting to develop theoretical approaches for explaining media choice and usage.

According to Tanis and Postmes (2003), some of the most significant turning points in human civilization have been marked by technological innovations that have increased our ability to store, transport and communicate information and knowledge. Modern communication technologies that rely on the processing power of computers challenge conventional notions of media and its use.

Barnes (2003) acknowledges that during the past twenty years, new communication technologies are being widely integrated into teaching and learning. These modern communication technologies broaden the scope of communication and open up communication possibilities not otherwise possible. Among some of these possibilities is efficiency and spend of communication, coordination with and control of multiple persons, ability to sort, send and retrieve messages at any time and place.

In educational environment, for instance, e-mail provides a new method for students and teachers to correspond with each other; it also allows electronic distribution of course material. Discussion lists can be used to support in-class conversation or provide distance education. Students around the world can come to talk about academic materials and assignments. It can also be used as a virtual classroom to bring together students both locally and globally. The Web has also emerged as a popular research tool for both teachers and students.

Bates (1995) noted that internet is one of the teachers and learners who are using it. E-mail is one type of communicating that allows for both one-to-one and one-to- many textual communications without regard to an individual's physical location.

Although many new communication technologies described as 'lean', and thus unsuitable for equivocal problem solving, research has also demonstrated that such technologies can be more effective than face-to-face channels. In fact, despite their commonly asynchronous nature, some organizations have reported positive outcomes when using them, including reduced delays in information exchange, improve maintenance of records and information received, increase coordination of geographic dispersed groups, and improving users' capabilities to process large amounts of information (Baltes et al. 2002).

On the other hand, Researchers found that these technologies usually do not incorporate all of the elements present in the Face to face communication. In other word, in spite of the benefits that new communication channels can offer, it is a limited symbolic representation system. Social and contextual cues that usually regulate and influence human communication dynamics are missing or attenuated.

Some researcher illustrate that face-to-face communication possesses inherent characteristics that make it more appropriate than other communication channels, that suppress too many of the Face to face communication elements (Short, Williams and Christie, 1976). The common explanation is that new channels, in contrast to face-to-face communication, lacks nonverbal cues and that, therefore, the relational tone of communication tends to be impersonal, less friendly, less emotional and more task-oriented.

The impact of new communication technologies in human communication, especially in education, has been the target of intense research. Communication in interaction between academic staff and students plays a key role in ensuring that they can accomplish their objectives at both educational and interpersonal relationship. Because of their job, academic staffs usually spend a lot of time in interaction with their students. Barnes (2003) proposed that the need to understand the impact of new technologies on education is growing as technological advances offer more communication options. In recent years, while many academic staffs have choice to turn to new communication channels as a means of communicating with students, few researches has been aimed to asses how academic staffs communicate with their students or through which channels? Or which channels they most used in interacting with their students? Whether new communication channels or traditional ones?

\subsection{Research Question}

Theoretically, the above dichotomies of communications channels (traditional channels vs. new communication channels) have some differences in terms of the nature of channels, characteristics of each channel and so on. Base on these differences, this study try to answer the following questions:

R.Q1- What are the level of academic staffs' experience (use) of each communication channel in faculty- student interaction?

R.Q2- What is academic staffs' perception of media richness factors?

R.Q3- Which communication channels do the academic staffs most frequently used for special message (based on characteristic of message)? 
R.Q4- Which communication channels most frequently used by the academic staffs based on type of relationship (Educational/ personal relationship)?

\subsection{Conceptual Framework}

For the purpose of this paper, we chose to investigate the model shown below. Based on this model that was derived from media richness theory and social presence theory, three factors could affect the selection of communication channels in order to interact with each other.

Based on the above mentioned theories, type of task refers to the nature of message that contains simple straightforward message (.e.g. clear message), Ambiguous message (e.g. messages that require discussion, negotiation or clarification) and Complex message (e.g. messages that require elaboration, explanation or demonstration with examples).

Type of interaction refers to the kind of relationship between academic staffs and their Students, include of task-focused (e.g. educational relationship) and relation-focused (personal relationship).

Richness of media means the ability of media to carry both verbal cues (, e.g. volume, tone and rhythm of sound) and nonverbal cues (e.g. gestures, facial expression and eye contact.), provides rapid feedback, convey personality traits and support the use of natural language.

Communication channels refer to both traditional and new channels of communication including face-to-face, telephone, mobile, Email, Written Message and online communication. In order to measure the level of academic staff's experiences (use) of each communication channel in interaction with their students, they were on the scale from 1-very low, 2- low, 3- Middle, 4- high and 5- very high.

\subsection{Hypotheses}

Based on conceptual framework, there are three factors that can influence the choice of communication channel by academic staffs. Two hypotheses were developed to explore these factors. The first hypothesis is related to the experience of academic staffs with different channels:

H1-Whether experience level differed across different communication channels.

H2-Role of academic staffs' perceptions (in terms of richness) in determining which different communication channels are used for interact with their students. The effectiveness of different constructs such as providing immediate feedback, conveying personality traits and carrying both verbal and nonverbal cues were examined in this study. (Noticed that the differences between the first three channels most used by the academic staffs were measured and the other three remaining with lower usage were left out).

H2a - There is significant relationship between the first communication channel most used by academic staffs and their perception of media richness.

H2b- There is significant relationship between the second communication channel most used by academic staffs and their perception of media richness.

H2c- There is significant relationship between the third communication channel most used by academic staffs and their perception of media richness.

\section{Literature review}

When two or more parsons work together, they have to communicate through some medium. While face-to-face was most commonly used medium in communicating, it's seems the dominance of face-to-face communication is changing. This means with the growth of communication technology and advances in network channels, face-to-face meetings are no longer the unique communication medium used by individuals. However, the degree to which media affect communication can change the way in which individuals choose media to communicate and can lead to better or worse relationship.

Although there has been a vast amount of literature investigating new communication channels, it is still not well understood how these new media are integrated into human communication behavior or which traditional media are replaced, if so, by the new communication channels. To answer these questions there has been research in the many dimensions of new communication media usage including: changing perceptions of communication media (Schement \& Stout, 1989); the technical and social characteristics of the new media (Huang \& Wei, 2000); the human conceptualization of the underlying properties, roles and functions of the new media (Katz \& Rice, 2002); the perceived characteristics of the new media (Trevino et al., 1990); the affect of context and social influence on the adoption and usage of the new media (Carlson \& Zmud, 1999).

In addressing the effectiveness of Communication technologies in interacting between academic staffs and their students, this research project joins a body of literature that aims to extend two of the most widely investigated media choice theories: Media Richness Theory (MRT) and Social Presence Theory (SPT). 


\subsection{Social Presence Theory}

According to Short, Williams, and Christie (1976), social presence is a subjective quality of the communication medium and relates to the social psychology concepts of intimacy (determined by physical distance, eye contact, smiling, and personal topics of conversation) and immediacy (determined by the medium's capacity in transmitting information). Tu (2002) has argued that social presence can be defined in terms of a combination of social relationships, communication styles, task analyses, feedback levels and measures of immediacy.

Short, Williams and Christie proposed the social presence theory at a time when the Internet as we know it today was yet to be conceptualized, let alone implemented. In spite of that, the theory has influenced much CMC research over the years.

They see social presence as the ability of individuals to collaborate effectively through technology, even when they are located in different locations and time frames. Social presence refers to the degree to which a medium allows communicators to experience others as being psychologically present, or the degree to which a medium is perceived to convey the actual presence of the communicators. Social presence can be a function of both verbal cues (e.g., tone of voice) and nonverbal cues (e.g., facial expression, direction of gaze, posture, and dress).

Short, Williams and Christie (1976) surveyed the literature on mediated communication and concluded that communication media differ in their ability to provide a sense of social presence. They also concluded that most new media are lacking in social presence. In other word, communicating by media is rather different than communicating in person. This has the implication that understanding may be distorted due to a lack of social cues and users tended to misinterpret messages. In addition, reduced social presence may lead to less emotionality in exchanges, weakening the interpersonal function of communication.

This theory classifies different communication media along a one-dimensional continuum of "social presence.” Media that are capable of providing a greater sense of intimacy and immediacy will be perceived as having a higher social presence. On a continuum of social presence, communication media such as FtF meetings, which are capable of conveying nonverbal and social context cues, is considered to have the most social presence, whereas CMC, written, text-based communication have less because they lack nonverbal feedback cues.

According to the social presence theory, communication tasks differ in their requirements for social presence. The appropriateness of a medium for performing certain communication tasks is determined by the degree to which the medium's characteristics of social presence fit the requirements of the tasks. Tasks that require interpersonal skills, such as resolving conflicts or negotiation, demand high social presence, whereas tasks such as exchanging routine information are low in their social presence requirements. Media like Face to face and group meetings are more appropriate for performing tasks with high social presence requirements, whereas media such as e-mail, letters and memos are fit for low social presence tasks.

Although most empirical research on social presence theory has failed to provide consistent support in terms of the theory's ability to predict media choice, however some studies have shown that SPT is predictive of media choice. For instance, Rice (1993) used social presence theory to compare traditional and new media by analyzing data from six studies designed to examine the use and effects of new media. He found that due to the lack of social presence, both voice mail and e-mail were ranked lower in their overall task appropriateness than traditional face-to-face meetings, and e-mail was ranked even lower than voice mail in both overall appropriateness and for exchanging timely or confidential information.

\subsection{Media Richness Theory}

The media richness theory is suggested by Daft and Lengel in 1986, it is viewed as a refinement and extension of the social presence theory. According to Dennis and Kinney (1998) richness of a medium is based on its ability to process rich information. He proposed Media Richness theory (MRT), which hypothesizes on the information carrying capacity of media. This capacity is increased by the extent to which the medium meets four criteria as follows:

- Feedback Capability - the ability of the medium facilitate instantaneous feedback (synchronicity) and clarification of issues during engagements.

- Multiple Cues/Communication Channels Utilized - the range of cues, (including body language, voice inflection, physical representations) facilitated by the medium.

- Language Variety - the ability of the medium facilitate engagements involving both numbers and natural language.

- Personal Focus/Source - the ability of the medium to convey the personal feelings and emotions of communicating parties.

The media richness theory classifies communication media along a continuum of "richness," where richness is based on the ability of media to carry nonverbal cues, provide rapid feedback, convey personality traits and support the use of 
natural language. These criteria impact upon human understanding and frame of reference. As Lam (1998) claimed, media richness refers to the ability of the media to change human understanding, overcome different conceptual frames of reference or clarify ambiguous issues in a timely manner. Consequently, communication media possessing more features of the criteria would rank higher on the richness scale compared to one possessing less.

The media richness theory propose that Face to face communication is the richest medium, followed in order by telephone, written personal, CMC, written formal and then numerical formal media. Oral media, such as Face to face and telephone, are believed to be richer than written media because they provide opportunities for immediate feedback and can have multiple cues including kinesics, facial expression and tone of voice and uses natural language that is high in variety. Especially in face-to-face, participants enable to use varying modes of communication: words, vocal cues (e.g., voice inflection, sighs), nonverbal communication (e.g., gestures, touch), and written or drawn communication (e.g., paper, blackboards). These modes combine to transmit factual information about the task and social information about the personal characteristics of team members. Other media have lesser abilities to transmit these different forms of communication (Wright, 2000).

In media richness theory media were placed in continuum of richness, from low in richness to high in richness. Daft \& Lengel (1984 cited in Sevince and Ambra 2002) state that, media low in richness are suitable for facilitating discussion over simple topics, while media high in richness are suitable for complex organizational topics. They focused on 'traditional' communication media, such that face-to-face meetings were considered the richest media, while the leanest media were considered to be formal, unaddressed documents (such as memos). Media were placed on a continuum of information richness, suggesting that the richness property of each medium was fixed.

Media richness theory differentiates between lean and rich media by the number of cue systems within each medium. This approach suggests that because CMC is a lean channel, it is useful for simple or clear messages. CMC is also more efficient for communication that does not require coordinated interaction efforts. On the other hand, a richer medium should be used for information that is ambiguous, emphatic or emotional (Wright, 2000).

Ambiguous refers to equivocal, whereby the communicators face the problem of confusion because there are too many possible meanings in the message. When words or events are ambiguous or equivocal, people don't need more information but they need a context or framework to help them sort through the data. They need a filter to help them to screen out interpretations that would turn out to be counterproductive. Therefore, Face to face meeting is best for ambiguous messages as it provides rapid feedback.

Complex messages refer to the unpredictable human dimensions and emotional aspects of interactions. It includes those messages that require further explanation, elaboration or clarification. Complex messages are neither objective nor computational procedures that clearly instruct people what to do. According to Keil and Johnson, (2002), complexity is more subjective or perception-dependent than ambiguity.

Keil and Johnson (2002) noticed that CMC and written media can oversimplify complex problems because they do not provide a means to convey feedback or information concerning personal feelings. In addition, Kock (2004) found that the lack of nonverbal and social cues in CMC interaction reduces social regulation, leading to more relaxing feeling and occasional overly emotional interactions.

Media richness theory proposed at a time when the Internet as we know it today was yet to be conceptualized and this has lead to difficulties in trying to position such media on a scale of relative richness. However, if we attempt to assess an electronic medium such as email, based on the criteria for media richness we see that, email is not a rich medium and thus should not be used by for highly equivocal communications.

\subsection{Characteristics of E-mail}

Sproull (1991) pointed out that e-mail technologies share the following five characteristics that differentiate them socially from other communication technologies:

i. E-mail is asynchronous. Senders and receivers are not required to attend to the same communication at the same time. Asynchrony is not only a matter of personal convenience; it means communication across time and space.

ii. Email is fast. Messages can be transmitted in only seconds across a continent, or even around the world. Speed makes possible long-distance conversations. I

iii. Email is text based. Messages convey typographic characters and the text in electronic communication makes it useful for exchanging documents as well as messages.

Iv. Email has multiple-receiver addressability. The sender can transmit the message to more than one person. This attribute means that without respect to physical, temporal or social location, people can delegate work, collaborate, form new groups and make collective decisions. 
v. Email has built-in external memory, which is important for social memory. The contents of electronic messages can be stored and retrieved later by other group members who wish to trace the history of the project and learn about the group's act.

Moran and Hawisher (1997) theorised e-mail as a hybrid, drawing on practices of reading and of writing, while developing its own unique qualities. This new mode of electronic communication has created new textual practices and new social practices around its texts. In particular, e-mail is distinguished by its ease of retrieval, speed, absence of paralinguistic cues and asynchronicity. These assets come with decreased security and some interesting conflicts:

i. Its seemingly ephemeral, fleeting nature (like speech) with the fixity of print.

ii. Its illusion of intimacy, with its possibility of being endlessly "forwarded”.

iii. Its sense of communal "warmth” despite geographical distances or anonymity.

iv. Its playful informality and spontaneity (like speech) with its decontextualised audience (like print).

These paradoxes create a communicative space like no other, distinct from Face to face and pen-and-paper communication. It is a potentially intimate space shared only by the sender and receiver.

So in general, e-mail is classified as a relatively lean medium according to the four characteristics of richness. In using e-mail, one is not able to communicate through multiple cues; immediate feedback may or may not be possible depending on the availability and inclination of the communication partner. Besides that, e-mail is based on the required use of a computer and the written word; hence it is not generally viewed as a personal mode of communication. Use of much language variety is also limited in e-mail.

\section{Methodology}

This study employed survey research design by distributing questionnaires (self administered). The questionnaires were distributed among 103 academic staff at one Iranian higher learning institution. The population of this study was 140 academic staffs and based on Bartlett, Kotrlik, \& Higgins (2001) sampling table a total of 103 respondents were selected randomly from a total population of 103 academic staffs.

Research instrument for this research is questionnaire. The questionnaire is adapted from Lam (1998) and Chidambaram, E. M., \& Dag, H. O (1998). The reliability of the questionnaire examined by calculating the internal consistency of the scales using Cronbach's alpha. In this study, the Cronbach's alpha for 6 items of media richness was 0.89 that is statistically acceptable.

\section{Findings and analysis}

\subsection{Personal Information of Respondents}

Among the 103 respondents of this study, 70(68\%) were male and 33(32\%) were female academic staffs. Majority $67(65 \%)$ of the respondents were PHD in term of academic qualification. This is followed by the Master 36 (35\%).

(Table 1)

Most of respondents, 75.7\%, had more than 10 years of teaching experience. However, the mean for respondents’ level of teaching experience is 10.8 years; the minimum level of experience was 1 year and the maximum level was 50 years experience of lecturing. In addition, all of the academic staffs, 103 respondents, in faculty of Communication and Social science had some experiences as a Supervisor or member of Committee Supervisory for their students.

(Table 2)

\subsection{The level of experience (use) of Communication Channels}

To answer RQ1, responses to the six channel used were examined. Table 3 summarizes the overall level of experience of communication channels by academic staffs. Generally, Face-to-Face interaction (mean, 4.52) becomes the most dominant communication channel for academic staff in interaction with their students. This is followed in order telephone line (mean, 2.9), mobile phone (mean = 2.8), email (mean, 2.7), Written message (Mean, 2.3) and Online communication (mean, 1.9).

(Table 3)

\subsubsection{Testing hypothesis one}

Hypothesis 1 addressed whether the level of experience for most communication channels used by academic staffs, is significantly deferent with other communication channels. In order to find out this, a pair sample T-test is used to comparing the mean of most communication channels used by academic staffs one by one.

Only between the mean of face to face and the mean of others channels; there is significant difference of mean. (e.g. Face-to-Face and telephone line $t=10.26, p=.00$, face to face on mobile phone, $t=13.2, p=.00$ ). In other word, Hypothesis No. 1 was supported, suggesting that academic staff's experience levels differed significantly with different 
media. That means face-to-Face, as a first channel, is significantly more used by academic staffs comparing with others communication channels.

(Table 4)

\subsection{Communication Channel Used and Perception of media richness}

RQ2 asked academic staffs perception of media richness factors. In this study, the academic staffs were asked to provide reasons of why they choose special medium in order to communicating with their students. The respondents are agreed that the channel must convey personality traits of mine and students (Mean $=4.2$ ), provide immediate feedback. (Mean $=4.28$ ), enable both me and students to use friendly language (Mean $=4.01$ ), carry both verbal and nonverbal cues $($ Mean $=4.4 .19)$, carry sufficient verbal cues $($ Mean $=4.28)$.

\section{(Table 5)}

Hypothesis 2.a through 2.c addressed whether there was a relationship between communication channel most used by academic staffs and their perception of media richness. As table 3 and 4 show, academic staffs chose Face-to-Face channel as their most used communication channel, significantly. This is followed by Telephone line and Email as the second and third one. In line with this, Pearson Product Moment Correlation was used to measure whether there is significant relationship between Face-to-Face, telephone line and Email, as the three most channels used by academic staff, and their perception of media richness. In other words, to understand whether the choice of Face-to-Face meetings or Telephone line and Email as the most used communication channel was due to their perception of media richness provided by this medium.

The results showed that, two of the above hypotheses were supported and there were significant relationship between the Face-to-face and Telephone line as channels and their perceptions of media richness. In other words, these results confirm that academic staffs preferred to use Face-to-Face and Telephone line channel due to the high level of social presence and media richness provided by these media. But, third hypothesis (relationship between Email and academic staffs' perception of media richness) was rejected. One possible reason is it's less experienced by academic staffs so that they couldn't consider Email as a rich medium. Or maybe they believed that Email comparing to Face-to-face and Telephone line is less effective because of its accessibility, cost, etc.

(Table 6)

Thus, perceptions of media richness play and important role for academic staffs in the selection of channels in order to interact with their students. This finding is also consistent with the social presence, which claims that Face-to-Face has the highest level of social presence and richness of information because it allows the simultaneous observation of multiple cues, including kinesics, facial expression and tone of voice. Face-to-Face also provides immediate feedback, personal interaction and uses of natural language that is high in variety.

\subsection{Communication Channel most used based on characteristics of message}

The result showed that 71(68.9\%) of the academic staffs preferred to see students Face-to-Face even to communicate straightforward and clear messages. Mobile phone was ranked as the second preferred medium 13(12.6\%), followed by telephone line 13(12.6\%), written message 3(2.9\%) and written message 3(2.9\%). So, this finding is contradicted with the notion of social presence and media richness theory, which propose that communicators would use a lean channel for straightforward and clear messages. One possible reason for why academic staffs were preferred to use Face-to-Face for almost every task is the phase that "old habits are hard to break". In other words, it grows into a habit to use this way of communication traditionally and culturally. Also, because the new communication technologies are more or less new arrival, maybe they feel some difficulties in terms of accessibility (for both side; sender and receiver), effectiveness and their ability to carrying message correctly.

\section{(Table 7)}

To communicate ambiguous messages, $78.6 \%$ of the academic staffs preferred to use Face to face meeting; only $21.4 \%$ chose to use Telephone line. This finding is consistent with the social presence and media richness theory, which suggested that a rich medium would be useful for ambiguous messages. There were $86.4 \%$ of academic staffs that chose Face-to-face as the preferred channel to communicate complex messages. It was followed by telephone line (9.7\%) and e-mail (3.9\%).

As mentioned earlier, these findings cast some doubt on media richness theory which states that task with different information rich requirements will require the use of different media. In this study, while media use differed within tasks, it did not differ across tasks. For example academic staffs appeared to be relying on traditional media for almost every task.

However, when this result is considered in light of the support for hypothesis No.1, a possible explanation emerges. Academic staffs' experiences with different media differ; they are more experienced with traditional media (e.g. face to 
face) and less experienced with new communication channels (e.g. online communication). As their level of experience with a channels increases, their use of that medium also tend to increase.

\subsection{Communication Channel Used based on Types of Interaction}

For both Educational and personal type of interactions, in general, most of the academic staffs preferred to use Face-to-Face channel for communicating with their students, followed by Mobile Phone and Email. Although, Face-to-Face and Telephone can consider as high level of media richness, however, According to the social presence and media richness theory, Email has lower level of social presence and media richness comparing to telephone. But, this study found that email as a second preferred channel by academic staffs. This preference of students could be explained by some characteristics of e-mail suggested by Sproull (1991), where he pointed out that e-mail technologies share the following five characteristics that differentiate them socially from other communication technologies: 1Email is asynchronous, 2- Email is fast, 3- Email is text based, 4- Email has multiple-receiver addressability and 5Email has built-in external memory, which is important for social memory.

(Table 8)

\section{Conclusion}

The major conclusion of this research is the perception of media richness play a key role in the selection of channels. As it was discovered in this study, Face-to-Face communication is the most used and preferred communication channel (mean=4.52) by academic staffs in interaction with their students followed by Telephone line (mean=2.9) and Email (mean=2.8). It also discovered that there is significant difference (H1) between the levels of experienced by academic staffs across the different channels.

This finding resonates with Chidambaram, et al (1998) which states that even though there is the existence of new communication technologies such as the internet which offers faster and cheaper facilities, traditional channels still remain most preferred channels among staffs of organization.

The main reason for this finding as supported by H2.a and H2.b is, because of their higher level of social presence and richness of information $(\mathrm{F}-\mathrm{F}=\mathrm{r}, .366, \mathrm{p}=.001 \&$ Telephone line $=\mathrm{r}, .349, \mathrm{p}=001)$. In other words, Face-to-Face and Telephone Line are chosen because academic staffs have experience with it, consider it to more effective and a rich medium and are generally satisfied with it. Nevertheless that the H2.c for the third communication channels used was rejected.

However, while Face-to-Face remains highly appropriate and popular in most situations, modern communication channels such as email can also provide a preferable solution in other contexts. Despite the lower usage of e-mail for ambiguous and complex messages, e-mail is found to be the third widely adopted and preferred communication medium among faculty members in faculty-student interaction, especially for sending Simple Messages and Complex Messages.

Another explanation for the results of this study may be is the factor of time. The element of time as discussed by the Social Information Processing Theory (SIP) is one of the most important factors in the decision making to use which kind of media in order to interacting which others (Whalter, 1996). These are because, while the multiple channels and cues available in face to face interaction expedited the exchange of information and fulfilling the task, the process slowed by the new communication channels.

In view of this, the principle of interactivity was developed by Burgoon et al. (2002). According to the principle of interactivity, the differences among channels are not just based on the number and types of cues filtered out, but also based on several criteria or structural affordances such as contingency, transformation, participation, proximity, synchronicity, parallelism and so on.

Thus, future study should consider examining the factor of time in selecting of media channels in order to interact with each other. Future studies also need to be conducted in order to determine the effect of principle of interactivity in each communication channels.

\section{References}

Baltes, B.B., Dickson, M.W, Sherman, M.P., Bauer, C.C. \& LaGanke, J.S. (2002). Computer mediated Communication and Group Decision Making: A meta analysis. Organizational Behavior and Human Decision Processes, 87(1), 156-179.

Barnes, S. B. (2003). Computer-mediated communication: Human-to-human communication across internet. Boston: Pearson Education.

Bates, T. (1995). Technology, open learning and distant education. New York, NY: Routledge.

Burgoon, J. K., Bonito, J. A., Ramirze, A., Dunbar, N. E., Kam, K. \& Fischer, J. (2002). Testing the interactivity principle: Effect of mediation, propinquity, and verbal and nonverbal modalities in interpersonal interaction. Journal of Communication, 52(3), 657-678. 
Carlson. JR. \& Zmud. R.W. (1999). Channel Expansion Theory and the Experiential Nature of Media Richness Perceptions. Academy of Management Journal, 42, 130-240.

Chidambaram, l. Eric Moe, Carl \& Dag, H. O. (1998). A study of factors influencing media choice in Norwegian Organizations. European Journal of Technology, 2, 102-113.

Craig, T., Harris, L., \& Smith, R. (1998). Rhetoric of the “contact zone”: Composition on the front lines. In T. Taylor \& I. Ward (Eds.), Literacy theory in the age of Internet. (pp. 122-145). New York: Columbia University Press.

Daft, R.L., \& Lengal, R.H. (1986). Organizational Information Requirements, Media Richness, and Structural Design. Management Science, 32(5), 554-571.

Dennies, A., \& Kinney, S. (1998). Re-evolution Media Richness: Cues, Feedback, and Task. IEEE Computer Society Press, 4, 21-30.

Doherty, C., \& Mayer, D. (2003). E-mail as a “contact zone” for teacher-student relationships. Journal of Adolescent and Adult Literacy, 4, 592-600.

Huang, W. W., \& Wei, K. K. (2000). An empirical investigation of the effects of group support system (GSS) and task type on group interactions from an influence perspective. Journal of Management Information Systems, 17(2), $181-206$.

Keil, M., \& Johnson, R. D. (2002). Feedback channels: Using social presence theory to compare voice mail to e-mail. Journal of Information System Education, 13(4), 295- 303.

Kock, N. (2004). The psychobiological model: Towards a new theory of computer-mediated communication based on Darwin evolutionn. Organization Science, 15(3), 327-349.

Lam, C. (1998). Communication satisfaction in relation to managerial roles and the choice of communication media. Unpublished master's thesis, University of Hong Kong, Hong Kong.

McCollough, M., \& Gremler, D. (1999). Guaranteeing student satisfaction: An exercise in treating students as customers. Journal of Marketing Education, 21, 18-130.

Naing, L., Winn, T., \& Rusli, B.N. (2006). Practical issues in calculating the sample size for prevalence studies, 1, 9-14.

Rice, R. E. (1993). Media appropriateness: Using social presence theory to compare traditional and new organizational media. Human Communication Research, 4, 451-484.

Short, J., Williams, E., \& Christie, B. (1976). The Social Psychology of Telecommunications. London: John Wiley and Sons.

Sproull, L., \& Goodman, L. (1991). Technology and Organizations. U.S: Pearson Education.

Tao, L. Q. (2002). Email: Instructional potentials and learning opportunities. Reading \& Writing Quarterly, 18, 285-288.

$\mathrm{Tu}, \mathrm{C}$. (2002). The measurement of social presence in an online learning environment. International Journal of E-Learning, 1(2), 34-45.

Walther, J. B. (1996). Computer-mediated communication: Impersonal, interpersonal and hyper personal interaction. Communication Research, 23(1), 3-34.

Wright, K. (2000). Computer-mediated social support, older adults and coping. Journal of Communication, 50(1), 100-118.

Zolten, J. (November, 1997). E-mail bonding: Making the most of electronic communication between teacher and student. Paper presented at the 83rd annual meeting of the National Communication Association, Chicago.

Table 1. Demographic of Respondents

\begin{tabular}{lll}
\hline Gender & Frequency & Percent \\
\hline Male & 70 & 68 \\
Female & 33 & 32 \\
& & \\
\hline Academic Qualification & Number & Percentage \\
\hline PHD & 67 & 65 \\
Master & 36 & 35 \\
\hline Total & 103 & 100.0 \\
\hline
\end{tabular}


Table 2. Experience of Lecture

\begin{tabular}{lrr}
\hline Experience of Lecture & Frequency & Percent \\
\hline $1-10$ & 25 & 24.3 \\
$11-20$ & 39 & 37.9 \\
$21-30$ & 30 & 29.2 \\
More than 31 & 8 & 7.8 \\
& & Percent \\
\hline The level of Supervision & 6 & 5.8 \\
\hline Bachelor & 41 & 39.8 \\
Master & 12 & 11.7 \\
PHD & 33 & 32.1 \\
All & 8 & 7.8 \\
Master \& Bachelor & 3 & 2.9 \\
Master \& PHD & 103 & 100 \\
\hline Total & & 3 \\
\hline
\end{tabular}

Table 3. The level of experience

\begin{tabular}{lrr}
\hline Cannels & Mean & Sd. Deviation \\
\hline Face-to- Face & 4.52 & .86 \\
Email & 2.8 & 1.50 \\
Mobile phone & 2.7 & 1.3 \\
Telephone line & 2.9 & 1.3 \\
Written Message & 2.3 & 1.3 \\
Online Communication & 1.9 & 1.1 \\
\hline
\end{tabular}

The channels were on scale from 1- very low to 5- very high.

Table 4. Pair sample T-test

\begin{tabular}{lrrr}
\hline Pair sample T-test & T-value & df & P-value \\
\hline Face-to- Face telephone line & 10.26 & 100 & 0.000 \\
Face-to- Face Mobile phone & 13.20 & 101 & 0.000 \\
Face-to- Face Email & 11.10 & 101 & 0.000 \\
\hline
\end{tabular}

Table 5. Academic staff's perception of media richness

Factor of media richness

Mean

Sd.

The channel must convey personality traits of mine and students,

e.g. friendliness, respectfulness and concern.

The channel must provide immediate feedback.

The channel must enable both me and students to use friendly

Language.

The channel must carry both verbal and nonverbal cues,

e.g. volume and eye contact.

The channel must carry sufficient verbal cues, e.g.

volume, tone and rhythm of sound.

The channel must carry sufficient nonverbal cues, e.g. gestures, facial expression and eye contact. 
Table 6. Pearson Correlation for Communication channels used and Perception of Richness of media

\begin{tabular}{lll}
\hline \multicolumn{3}{c}{ Media richness } \\
\hline Channel & r & p-value \\
\hline Face-to-face & .366 & ${ }^{*} .001$ \\
Telephone Line & .349 & .001 \\
Email & .450 & .067
\end{tabular}

*Correlation is significant at the 0.01 level.

Table 7. Communication Channel used based on characteristics of Message

\begin{tabular}{lrrr}
\hline $\begin{array}{l}\text { Characteristics of } \\
\text { Message }\end{array}$ & Communication Channels & Frequency & Percentage \\
\hline Simple Message & Face-to-Face & 71 & 68.9 \\
& Mobile Phone & 13 & 12.6 \\
& Telephone line & 13 & 12.6 \\
& Email & 3 & 2.9 \\
& Written Message & 3 & 2.9 \\
\hline \multirow{3}{*}{ Ambiguous Message } & Face-to-Face & 81 & 78.6 \\
& Telephone Line & 22 & 21.4 \\
\hline Complex Message & Face-to-Face & 89 & 86.4 \\
& Telephone line & 10 & 9.7 \\
& Email & 4 & 3.9 \\
\hline
\end{tabular}

Table 8. Communication Channel Used and Types of Interaction

\begin{tabular}{llrr}
\hline Educational relationship & Communication Channels & frequency & Percentage \\
\hline To fix appointments with students & Mobile Phone & 42 & 40.8 \\
to see them or do something. & Face-to-Face & 29 & 28.2 \\
& Telephone Line & 19 & 18.4 \\
& Written Message & 12 & 10.7 \\
\hline To provide feedback on students' & Face-to-Face & 40 & 38.8 \\
assignments or final year projects. & Telephone Line & 25 & 24.3 \\
& Mobile Phone & 25 & 24.3 \\
\hline To pass lecture or tutorial notes to & Face-to-Face & 88 & 85.4 \\
students. & Email & 15 & 14.6 \\
\hline To encourage and motivate & Face-to-Face & 80 & 77.7 \\
students to do something. & Email & 11 & 10.7 \\
& Mobile Phone & 6 & 5.8 \\
\hline Personal relation ship & Channels & 73 & \\
\hline To show concern about students' & Face to Face & & Percentage \\
problems (e.g. sympathy, & & 14.9 \\
condolence). & & 11 & \\
& Email & 54 & 13.6 \\
& Mobile Phone & & 52.4 \\
\hline To invite students to eat/ drink & Face to face & 31 & 30.1 \\
something with you. & & 8 & 7.8 \\
& Mobile phone & 6 & 5.8 \\
\hline
\end{tabular}




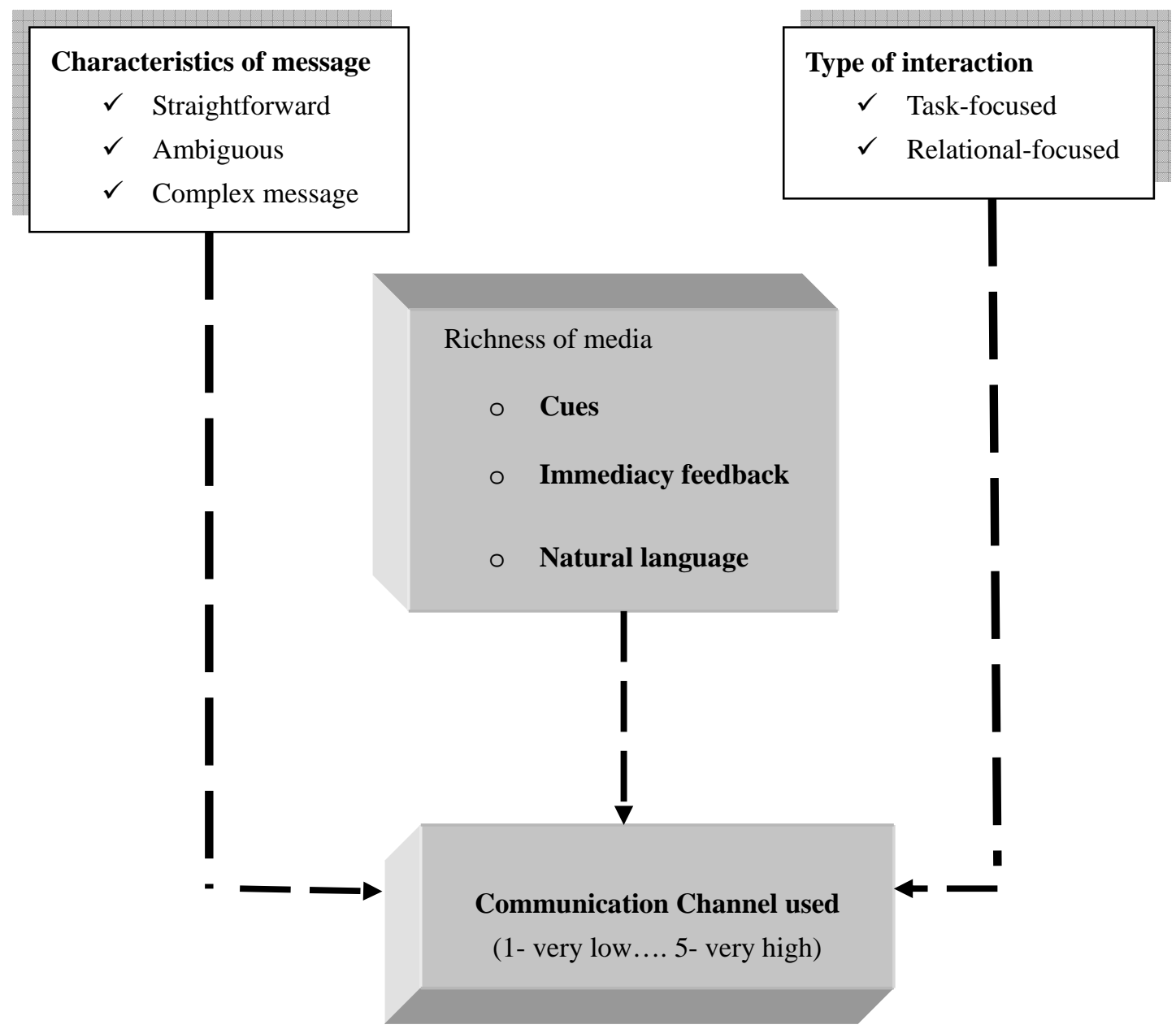

Figure 1. Conceptual Framework 